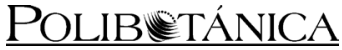

Núm. 40, pp. 29-44; México, 2015

DOI: $10.18387 /$ polibotanica. 40.2

\title{
COMPOSICIÓN TAXONÓMICA Y DATOS ECOLÓGICOS DE LAS LICOFITAS Y MONILOFITAS DE LA SIERRA DE TAMAULIPAS, TAMAULIPAS, MÉXICO
}

\section{TAXONOMIC COMPOSITION AND ECOLOGICAL DATA OF THE LYCOPHYTES AND MONILOPHYTES FROM SIERRA DE TAMAULIPAS, TAMAULIPAS, MEXICO}

\author{
Ana María Hernández-Mendoza ${ }^{1}$, María de la Luz Arreguín-Sánchez ${ }^{2}$, \\ Jesús García-Jiménez ${ }^{\mathbf{1}}$, y María Concepción Herrera-Monsivais ${ }^{\mathbf{1}}$ \\ ${ }^{1}$ Instituto Tecnológico de Ciudad Victoria, Tamaulipas. Blvd. Emilio Portes Gil núm. 1301, \\ CP 87010, AP175. Cd. Victoria, Tamaulipas, México. ${ }^{2}$ Escuela Nacional de Ciencias Bio- \\ lógicas, Instituto Politécnico Nacional. Prolongación de Carpio y Plan de Ayala, \\ col. Santo Tomás, CP11340, México, DF y Becaria de COFAA del IPN.
}

\section{RESUMEN}

En el presente estudio se enlistan las licofitas y monilofitas que habitan en la sierra de Tamaulipas, registrándose un total de 18 familias, 38 géneros, 91 especies y ocho variedades. La familia Pteridaceae es la más representativa tanto en géneros (13) como en especies (36), siendo el género Cheilanthes el más sobresaliente, además se registran por primera vez 23 taxones para el estado de Tamaulipas. Se recolectó una especie que se consideraba había desaparecido de la zona como es el caso de Schaffneria nigripes Fée, que desde hace 30 años no se había vuelto a encontrar. El único taxón endémico para el estado fue Notholaena brevistipes Mickel. Los ejemplares que se mencionan en el trabajo como Anemia sp., Elaphoglossum sp. y Selaginella hansenii Hieron. vel. aff., Cheilanthes eatonii Baker vel. aff. y Ch. lozanoi (Maxon) R.M. Tryon \& A.F. Tryon podrían tratarse de nuevas especies. En este trabajo se incluye la distribución de las especies por tipos de vegetación, microhábitats y algunos datos ecológicos. Se identificaron cinco tipos de vegetación y tres asociaciones vegetales en el área de estudio.

Palabras clave: hábitat, distribución, tipos de vegetación, distribuciones altitudinales.

\section{ABSTRACT}

In the present study are recorded a total of 18 families, 38 genera, 91 species and eight varieties of lycophytes and monilophytes. The family Pteridaceae is the most representative with (13) genera and (36) species, being the genus Cheilanthes the best represented, also recorded for first time 23 taxa for the state of Tamaulipas. We collected Schaffneria nigripes Fée, a species that had not been seen more than 30 years ago in Tamaulipas. I was found as the only species endemic to Tamaulipas Notholaena brevistipes Mickel. The examples mentioned in this paper as Anemia sp., Elaphoglossum sp. and Selaginella han- 
senii Hieron. vel. aff., Cheilanthes eatonii Baker vel. aff. y Ch. lozanoi (Maxon) R.M. Tryon \& A.F. Tryon could be considered new species. This work includes the distribution of species by vegetation types, micro-habitats and some ecological data. We identified five vegetation types and three plant associations in the study area.

Key words: habitat, distribution, vegetation types, altitudinal distributions.

\section{INTRODUCCIÓN}

La sierra de Tamaulipas se encuentra ubicada en la parte sureste del estado de Tamaulipas, en los límites del Trópico de Cáncer. Se extiende a lo largo de un eje norte-sur por aproximadamente 100 kilómetros que incluye los municipios de González, Llera de Canales, Soto la Marina, Villa de Aldama y Villa de Casas. En el núcleo de la Sierra se levantan los picos de sierra Azul y cerro Picacho, con altitudes de 1400 y 1200 m.s.n.m., respectivamente (INEGI, 1983). La sierra de Tamaulipas es una zona de confluencia biogeográfica lo que permite que exista una gran variedad de especies con afinidades tropicales y boreales según lo indica Puig (1968), razón por la que es considerada como la Región Terrestre Prioritaria núm. 91 por la Comisión Nacional para el Conocimiento y Uso de la Biodiversidad (Arriaga et al., 2000). Actualmente está propuesta como Área Natural Protegida, por la Comisión Nacional de Áreas Naturales Protegidas (CONANP, 2006).

Entre los estudios que se han realizado para la región se encuentran el de Martín et al., (1954) quienes recolectaron en los bosques de pino-encino a Pteridium aquilinum (L.) Kuhn. Puig (1968) publicó notas relacionadas con la flora y vegetación de la sierra de Tamaulipas (México), distinguiendo seis tipos de vegetación: bosque tropical deciduo, bosque espinoso, matorral espinoso, estepa arbustiva espinosa, bosque de encino y bosque de pino, y en los últimos dos tipos de vegetación menciona la presencia de Pteridium aquilinum. Hernández-Mendoza (2007) realizó un estudio pteridológico en la parte sur de la sierra de Tamaulipas, encontró 11 familias, 33 géneros, 62 especies y seis variedades, de los cuales 10 de los taxones fueron nuevos registros para el estado, posteriormente, la misma autora en el año 2010 amplió el área de muestreo hacia la parte norte de la sierra de Tamaulipas, citando un total de 18 familias, 38 géneros, 91 especies y ocho variedades, de los cuales 23 entidades taxonómicas resultaron nuevos registros para el estado y cinco ejemplares que no fue posible su identificación por no coincidir los caracteres morfológicos con ninguna especie descrita. También se hace una lista en la tabla 1 de los sitios de colecta de estas especies en la sierra de Tamaulipas.

El objetivo principal de este trabajo fue dar a conocer los datos florísticos y ecológicos de las especies de licofitas y monilofitas que habitan en la sierra de Tamaulipas en los diferentes tipos de vegetación, además de aportar información de nuevos registros para el estado, datos relacionados a especies raras, endemismos y ejemplares que no fue posible su identificación hasta especie. 
Hernández-Mendoza, A.M. et al.; Composición taxonómica de licofitas y monilofitas, sierra Tamps., Tamps., Méx.

Tabla 1. Lista de sitios de colecta de licofitas y monilofitas de la sierra de Tamaulipas.

\begin{tabular}{|c|c|c|c|c|c|c|c|}
\hline \multirow[t]{2}{*}{ Localidades } & \multicolumn{6}{|c|}{ Coordenadas } & \multirow{2}{*}{$\begin{array}{l}\text { Altitud } \\
\text { m.s.n.m }\end{array}$} \\
\hline & \multicolumn{3}{|c|}{ Longitud W } & \multicolumn{3}{|c|}{ Latitud N } & \\
\hline Rancho San Miguel & $98^{\circ}$ & $20^{\circ}$ & $0.09 ”$ & $23^{\circ}$ & $10^{6}$ & $44.22 ”$ & 917 \\
\hline Rancho la Sauceda & $98^{\circ}$ & $20^{\circ}$ & $10.42 ”$ & $23^{\circ}$ & 06 & $22.4 ”$ & 674 \\
\hline Minas San Miguel & $98^{\circ}$ & $19^{6}$ & $42.68 ”$ & $23^{\circ}$ & $09^{\circ}$ & $40.84 ”$ & 923 \\
\hline El Pueblito & $98^{\circ}$ & $19^{6}$ & $41.53 ”$ & $23^{\circ}$ & 08 & $28.93 ”$ & 848 \\
\hline Las Pilas Santa María & $98^{\circ}$ & $22^{\circ}$ & $26.33 ”$ & $23^{\circ}$ & $10^{6}$ & $33.34 ”$ & 905 \\
\hline Ejido Torrecillas & $98^{\circ}$ & $22^{\circ}$ & $27.21 ”$ & $23^{\circ}$ & $08^{6}$ & $46.04 ”$ & 797 \\
\hline Soyatal & $98^{\circ}$ & $23^{6}$ & $2.10 "$ & $23^{\circ}$ & $09^{\circ}$ & $1.10 ”$ & 998 \\
\hline Ejido Eduardo Benavides & $98^{\circ}$ & $20^{\circ}$ & $43.71 ”$ & $23^{\circ}$ & $09^{\circ}$ & $23.57 ”$ & 800 \\
\hline El Olivo & $98^{\circ}$ & 17 & $31.15 ”$ & $22^{\circ}$ & 56 & $1.57 ”$ & 200 \\
\hline Cerro Ejido Torrecillas & $98^{\circ}$ & $22^{\circ}$ & $15.38 "$ & $23^{\circ}$ & $09^{\circ}$ & $35.61 ”$ & 1180 \\
\hline Riscos Torrecillas & $98^{\circ}$ & 22 ' & $7.71 \%$ & $23^{\circ}$ & 09 ' & $12.14 "$ & 920 \\
\hline Cerro San Miguel & $98^{\circ}$ & 19 ' & $50.20 ”$ & $23^{\circ}$ & $10^{\prime}$ & $10.17^{\prime \prime}$ & 1091 \\
\hline Cerro Pedregoso & $98^{\circ}$ & $21^{\prime}$ & $12.29 "$ & $23^{\circ}$ & $05^{\prime}$ & $54.39 ”$ & 892 \\
\hline Cerro Ejido Eduardo Benavides & $98^{\circ}$ & 21, & $11.45^{\prime}$ & $23^{\circ}$ & $08^{\prime}$ & $49.60 "$ & 1000 \\
\hline Cantiles Torrecillas & $98^{\circ}$ & $23^{\prime}$ & $23.70 "$ & $23^{\circ}$ & $10^{\prime}$ & $8.76^{\prime \prime}$ & 605 \\
\hline Arroyo San Miguel (Izquierda) & $98^{\circ}$ & 20 ' & $31.22 ”$ & $23^{\circ}$ & $10^{\prime}$ & $52.26 "$ & 893 \\
\hline Arroyo San Miguel & $98^{\circ}$ & 20 & $5.81 ”$ & $23^{\circ}$ & $10^{\prime}$ & $48.25 "$ & 925 \\
\hline Ejido Santa María & $98^{\circ}$ & 21, & $23.48 ”$ & $23^{\circ}$ & 12 ' & $52.37^{\prime \prime}$ & 1056 \\
\hline San Antonio & $98^{\circ}$ & $24^{\prime}$ & $26.28 "$ & $23^{\circ}$ & 04 & $21.21 ”$ & 345 \\
\hline Ejido El Sabinito & $98^{\circ}$ & 23 , & $42.30 "$ & $23^{\circ}$ & 37 & $41.11 ”$ & 355 \\
\hline Carretera vieja Victoria Soto & & & & & & & \\
\hline la Marina Km 85-86 & $98^{\circ}$ & $25^{\prime}$ & $56.62 ”$ & $23^{\circ}$ & $34^{\prime}$ & $33.67 ”$ & 485 \\
\hline Francisco Castellanos & $98^{\circ}$ & 32 ' & $23.65 "$ & $23^{\circ}$ & $17^{\prime}$ & $29.92 ”$ & 519 \\
\hline Camino a San José & $98^{\circ}$ & $28^{\prime}$ & $8.99 ”$ & $23^{\circ}$ & $18^{\prime}$ & $48.92 ”$ & 1029 \\
\hline Valle San José & $98^{\circ}$ & $26^{\prime}$ & $46.14 ”$ & $23^{\circ}$ & $19^{\prime}$ & $22.61 ”$ & 893 \\
\hline Rancho Corazón de Jesús Punt.48 & $98^{\circ}$ & $23^{\prime}$ & $29.59 "$ & $23^{\circ}$ & $18^{\prime}$ & $28.45^{\prime \prime}$ & 930 \\
\hline Ejido Chijol & $98^{\circ}$ & 27 & $15.34 ”$ & $23^{\circ}$ & 12 ' & $52.14 ”$ & 943 \\
\hline Eslabones & $98^{\circ}$ & 15 , & $5.97 ”$ & $23^{\circ}$ & 25, & $32.70 "$ & 250 \\
\hline Ejido La Esperanza & $98^{\circ}$ & 21 , & $19.92 ”$ & $23^{\circ}$ & $37^{\prime}$ & $14.20 ”$ & 187 \\
\hline Rancho el Picacho & $98^{\circ}$ & $33^{\prime}$ & $36.33 ”$ & $23^{\circ}$ & $23^{\prime}$ & $48.77^{\prime \prime}$ & 570 \\
\hline El Puerto (Rancho El Picacho) 1 & $98^{\circ}$ & $32^{\prime}$ & $50.02 ”$ & $23^{\circ}$ & 24 & $43.73 ”$ & 857 \\
\hline El Puerto (Rancho El Picacho) 2 & $98^{\circ}$ & $34^{\prime}$ & $35.99 "$ & $23^{\circ}$ & $16^{\prime}$ & $20.12 ”$ & 437 \\
\hline San José (Cañón del Diablo) 1 & $98^{\circ}$ & $25^{\prime}$ & $38.03 ”$ & $23^{\circ}$ & $17^{\prime}$ & $47.71 ”$ & 767 \\
\hline San José (Cañón del Diablo) 2 & $98^{\circ}$ & $25^{\prime}$ & $17.71 ”$ & $23^{\circ}$ & $17^{\prime}$ & $40.96 "$ & 789 \\
\hline San José (Cañón del Diablo) 3 & $98^{\circ}$ & $25^{\prime}$ & $15.90 "$ & $23^{\circ}$ & $17^{\prime}$ & $44.86 "$ & 909 \\
\hline El Plomo & $98^{\circ}$ & $13{ }^{\prime}$ & $45.64 ”$ & $23^{\circ}$ & 08 & $11.76 "$ & 556 \\
\hline Rancho Briones & $98^{\circ}$ & $14{ }^{\prime}$ & $57.97 ”$ & $23^{\circ}$ & 09 ' & $26.84 ”$ & 918 \\
\hline La Lobera & $98^{\circ}$ & $15^{\prime}$ & $6.05 \%$ & $23^{\circ}$ & $25^{\prime}$ & $30.75^{\prime \prime}$ & 224 \\
\hline Rancho El Tigre & $98^{\circ}$ & $17^{\prime}$ & $54.56 ”$ & $23^{\circ}$ & $18^{\prime}$ & $48.92 ”$ & 306 \\
\hline Rancho Los Venados-Valle & $98^{\circ}$ & $28^{\prime}$ & $50.89 ”$ & $23^{\circ}$ & 24 & $39.65 "$ & 422 \\
\hline Camino Rancho Los Venados-Valle & $98^{\circ}$ & 22 & $9.15 \%$ & $23^{\circ}$ & 21, & $12.29 ”$ & 748 \\
\hline Cerro La Ventana & $98^{\circ}$ & $25^{\prime}$ & $58.94 ”$ & $23^{\circ}$ & $09^{\prime}$ & $1.76 "$ & 940 \\
\hline Ejido Las Yucas & $98^{\circ}$ & 08 & $28.40 ”$ & $23^{\circ}$ & 14, & $0.23 ”$ & 319 \\
\hline El Tuliyo (Ej. El Chijol) 1 & $98^{\circ}$ & $28^{\prime}$ & $17.85 "$ & $23^{\circ}$ & $12^{\prime}$ & $46.79 ”$ & 594 \\
\hline El Tuliyo (Ej. El Chijol) 2 & $98^{\circ}$ & 28 & $18.15 "$ & $23^{\circ}$ & 05 , & 20.14" & 613 \\
\hline
\end{tabular}




\section{Método}

Se realizaron 33 salidas a la Sierra de Tamaulipas durante los periodos septiembre 2004 a enero 2006 y enero 2009 a marzo 2010 para recolectar material botánico (tabla 1). El criterio que se utilizó para definir los tipos de vegetación en campo fue el de Rzedowski (1978). Las determinaciones taxonómicas se realizaron de acuerdo con las obras de Mickel y Beitel (1988), Moran y Riba (1995) y Mickel y Smith (2004). La corroboración taxonómica se llevó a cabo con base en el material existente en el Herbario Jerzy Rzedowski y Graciela Calderón de la Escuela Nacional de Ciencias Biológicas del Instituto Politécnico Nacional (ENCB) y el material colectado se depositó en el Laboratorio de Botánica del Instituto Tecnológico de Ciudad Victoria (ITCV).

Se elaboraron dendrogramas resultado de una matriz de datos de presencia-ausencia y los análisis estadísticos se analizaron bajo el programa NTSYSpc, versión 2.02 .

\section{RESUlTAdos}

La pteridoflora de la sierra de Tamaulipas se encuentra conformada por 18 familias, 38 géneros y 91 especies (tabla 2). La familia Pteridaceae destaca por presentar un mayor número de géneros y especies con 13 y 36 especies respectivamente; el género Cheilanthes es el que tiene una mayor representación de especies, con un total de 13; lo contrario ocurre con Athyriaceae, Blechnaceae, Equisetaceae, Lycopodiaceae, Lygodiaceae, Nephrolepidaceae, Osmundaceae y Tectariaceae, que presentan un género y una especie, quedando consideradas en este estudio como familias mono-específicas.
Apéndice 1. Lista de licofitas y monilofitas presentes en la Sierra de Tamaulipas. El asterisco $(*)$ representa los nuevos registros para el estado. Los dos géneros citados como sp. y las tres especies como vel. aff. podrían tratarse de nuevas entidades taxonómicas. El criterio subclases, órdenes, familias y géneros se tomó de Christenhusz et al. (2011).

Subclase: Lycopodiidae

Orden: Lycopodiales

Familia: Lycopodiaceae

1. Lycopodiella cernua L.*

Orden: Selaginellales

Familia: Selaginellaceae

2. Selaginella delicatissima Linden ex A. Braun

3. Selaginella extensa Underw

4. Selaginella hansenii Hieron vel. aff.

5. Selaginella hoffmannii Hieron *

6. Selaginella lepidophylla (Hook. \& Grev.) Spring

7. Selaginella novoleonensis Hieron.

8. Selaginella pallescens (C. Presl) Spring 9. Selaginella stenophylla A. Braun 10. Selaginella tenella (P. Beauv.) Spring* 11. Selaginella wrightii Hieron.

Subclase: Equisetidae

Orden: Equisetales

Familia: Equisetaceae

12. Equisetum hyemale A. Braun ex Engelm. L. var. affine (Engelm.) A. A. Eaton

Subclase: Ophioglossidae

Orden: Psilotales

Familia: Psilotaceae

13. Psilotum complanatum Sw. * 14. Psilotum nudum (L.) P. Beauv. 
Subclase: Polypodiidae

Orden: Osmundales

Familia: Osmundaceae

15.-Osmunda regalis (L.) var. spectabilis

A. Gray*

Orden: Hymenophyllales

Familia: Hymenophyllaceae

16. Trichomanes hymenophylloides

Bosch*

17. Trichomanes reptans $S w .{ }^{*}$

Orden: Schizaeales

Familia: Lygodiaceae

18. Lygodium venustum $S w$.

Familia: Anemiaceae

19. Anemia sp.

20. Anemia adiantifolia (L.) Sw.

21. Anemia hirsuta (L.) Sw.

22. Anemia karwinskyana (C. Presl) Prant1*

23. Anemia mexicana Klotzsch var. mexicana

24. Anemia oblongifolia (Cav.) Sw. *

Orden: Polypodiales

Familia: Dennstaedtiaceae

25. Dennstaedtia globulifera (Poir.) Hieron.

26. Pteridium caudatum (L.) Maxon

Familia: Pteridaceae

27. Adiantum capillus-veneris $\mathrm{L}$.

28. Adiantum concinnum Humb. \& Bonpl. ex Willd.

29. Adiantum trapeziforme $\mathrm{L}$.

30. Adiantum tricholepis Fée

31. Adiantum tenerum Sw.

32. Vittaria graminifolia Kaulf.

33. Llavea cordifolia Lag.

34. Pteris biaurita L. *

35. Cheilanthes aemula Maxon

36. Cheilanthes angustifolia Kunth
37. Cheilanthes arizonica (Maxon) Mickel*

38. Cheilanthes bonariensis (Willd.)

Proctor

39. Cheilanthes brachypus (Kunze) Kunze*

40. Cheilanthes eatonii Baker vel. aff.

41. Cheilanthes farinosa (Forssk.) Kaulf. *

42. Cheilanthes kaulfussii Kunze

43. Cheilanthes leucopoda Link

44. Cheilanthes lozanoi (Maxon) R.M.

Tryon var. seemannii (Hook.) Mickel \& Beitel

45. Cheilanthes lozanoi (Maxon) R.M. Tryon \& A.F. Tryon vel. aff.

46. Cheilanthes microphylla (Sw.) Sw.

47. Cheilanthes tomentosa Link

48. Cheiloplecton rigidum (Sw.) Fée var. rigidum

49. Aspidiotis meifolia (D.C. Eaton) Pic.Serm.

50. Mildella fallax (M. Martens \& Galeotti) Nesom

51. Mildella intramarginalis (Kaulf. ex Link) Trevis.

52. Notholaena brevistipes Mickel

53. Notholaena candida (M. Martens \& Galeotti) Hook.

54. Astrolepis cochisensis (Goodd.) D.M. Benham \& Windham

55. Astrolepis crassifolia (T. Moore \& Houlston) D.M. Benham \& Windham

56. Astrolepis integerrima (Hook.) D.M. Benham \& Windham

57. Astrolepis sinuata (Lag. ex Sw.) D.M. Benham \& Windham

58. Pellaea ovata (Desv.) Weath.

59. Pellaea ternifolia (Cav.) Link.

60. Hemionitis palmata $\mathrm{L}$.

61. Bommeria elegans (Davenp.) Ranker \& Haufler*

62. Bommeria pedata (Sw.) E. Fourn. * 
Familia: Aspleniaceae

63. Asplenium cuspidatum Lam.

64. Asplenium minimum M. Martens \& Galeotti

65. Asplenium monanthes $\mathrm{L}$.

66. Asplenium praemorsum $\mathrm{Sw}$.

67. Asplenium pumilum $\mathrm{Sw}$.

68. Schaffneria nigripes Fée

Familia: Thelypteridaceae

69. Thelypteris concinna (Willd.) Ching*

70. Thelypteris hispidula (Decne) C.F. Reed

71. Thelypteris melanochlaena (C. Chr.)

C. F. Reed*

72. Thelypteris oligocarpa (Humb. \&

Bonpl. ex Willd.) Ching

73. Thelypteris ovata R. P. St. John var. lindheimeri (C. Chr.) A. R. Sm.

74. Thelypteris puberula (Baker) C.V. Morton var. puberula

Familia: Blechnaceae

75. Blechnum appendiculatum Willd.

Familia: Athyriaceae

76. Diplazium lonchophyllum Kunze*

Familia: Dryopteridaceae

77. Elaphoglossum sp.

78. Elaphoglossum sartorii (Liebm.) Mickel*

79. Ctenitis melanosticta (Kunze) Copel.

80. Phanerophlebia nobilis (Schltdl. \& Cham.) C. Presl

Familia: Nephrolepidaceae

81. Nephrolepis hirsutula (G. Forst.) C. Presl*

Familia: Tectariaceae

82. Tectaria heracleifolia (Willd.) Underw.
Familia: Polypodiaceae

83. Campyloneurum angustifolium (Sw.) Fée

84. Pleopeltis mexicana (Fée) Mickel \& Beitel

85. Polypodium hartwegianum Hook.

86. Polypodium madrense J. Sm. *

87. Polypodium plebeium Schltdl. \& Cham. 88. Polypodium polypodioides (L.) Watt var. michauxianum Weath.

89. Phlebodium areolatum (Humb. \& Bonpl. ex Willd.) J. Sm.

90. Phlebodium decumanum (Willd.) J. Sm. * 91. Pecluma plumula (Humb. \& Bonpl. ex Willd) M. G. Price

Los géneros más representativos en cuanto a especies son: Selaginella (10), Anemia (6), Thelypteris (6), Adiantum (5), Asplenium (5), Astrolepis (4) y Polypodium (4). Los géneros representados con una especie son: Aspidotis, Blechnum, Campyloneurum, Cheiloplecton, Ctenitis, Dennstaedtia, Diplazium, Equisetum, Hemionitis, Llavea, Lycopodiella, Lygodium, Nephrolepis, Osmunda, Pecluma, Phanerophlebia, Pleopeltis, Pteridium, Pteris, Schaffneria, Tectaria y Vittaria.

Como especie endémica para Tamaulipas se colectó a Notholaena brevistipes y como nuevos registros para la entidad federativa se tienen los siguientes: Anemia karwinskiana, A. oblongifolia, Bommeria pedata, B. elegans, Cheilanthes arizonica, Ch. brachypus, Ch. eatonii, Ch. farinosa, Diplazium lonchophyllum, Elaphoglossum sartorii, Lycopodiella cernua, Nephrolepis hirsutula, Osmunda regalis var. spectabilis, Phlebodium decumanum, Polypodium madrense, Psilotum complanatum, Pteris biaurirta, Selaginella hoffmannii, S. tenella, 
Hernández-Mendoza, A.M. et al.; Composición taxonómica de licofitas y monilofitas, sierra Tamps., Tamps., Méx.

Thelypteris concinna, T. melanochlaena, Trichomanes hymenophylloides y T. reptans.

Los tipos de vegetación presentes en la sierra de Tamaulipas según el criterio de Rzedows$\mathrm{ki}(1978)$ fueron las siguientes: bosque tropical subcaducifolio (BTSC), presente en las partes bajas y en cañadas de las zonas altas de la sierra; Palmar (PAL/BQ) en las partes medias y altas de la sierra en asociación con Quercus, matorral xerófilo (MX) en las partes bajas y en laderas de cerros, bosque de galería (BGALE) en las partes bajas y altas de la sierra sobre las orillas de ríos o arroyos, bosque de Pinus (BP) se encuentra en las zonas altas de la sierra, generalmente formando asociación con Quercus, bosque de Quercus (BQ) se localiza en la zona de estudio asociado con bosque tropical subcaducifolio; bosque de Pinus-Quercus (BPQ) considerada una comunidad diferente de los pinares o encinares, ya que al parecer cuando los pinos y encinos forman un bosque mixto presentan una flora particular (Rzedowski, 1978; Arreguín-Sánchez et al., 2009a), y aunque este tipo de vegetación no es considerada como tal en el trabajo de Rzedowski (1978), en la zona de estudio forman ecotonos que albergan una gran riqueza de licofitas y monilofitas.

\section{Distribución de especies por tipos de vegetación}

Bosque tropical subcaducifolio: se observaron 16 especies de las cuales solamente cuatro son características de este tipo de vegetación como Adiantum tricholepis, Cheiloplecton rigidum, Lygodium venustum, Schaffneria nigripes, colectados en las localidades de ejido San Andrés, ejido San Antonio municipio de González; Eslabones, La Lobera, ejido El Sabinito, Carretera vieja Victoria-Soto la Marina Km 85-86 municipio de Soto la Marina; rancho el Picacho, valle de San José, ejido Torrecillas municipio de Casas; Ejido el Plomo, ejido las Yucas municipio de Aldama.

Matorral xerófilo: se identificaron once especies, de las cuales Cheilanthes leucopoda y Selaginella extensa, prefieren únicamente este tipo de vegetación, colectadas en las localidades El Puerto, rancho Corazón de Jesús municipio de Casas.

Bosque de Quercus: en la zona de estudio se encontró este tipo de vegetación, en algunas localidades se aprecia una clara predominancia de encinos, y en otras áreas una mezcla de encinos con elementos de bosque tropical subcaducifolio. En los encinares con marcada predominacia de Quercus se distribuyen 34 especies de las que únicamente Astrolepis crassifolia y Elaphoglossum sartorii prefieren solamente este tipo de vegetación, los taxones fueron colectados en las localidades de Cerro Pedregoso municipio de González y en el Rancho Corazón de Jesús municipio de Casas.

\section{Bosque de Quercus con elementos de} bosque tropical subcaducifolio: en esta asociación vegetal se colectaron 25 especies, los taxones exclusivos de este tipo de vegetación son Diplazium lonchophyllum, Selaginella hoffmannii, S. pallescens y Trichomanes hymenophylloides, colectados en las localidades de Rancho Briones municipio de Aldama y Rancho Corazón de Jesús municipio de Casas.

Bosque de Pinus: en este tipo de vegetación las licofitas y monilofitas son escasas ya que solamente se registraron nueve especies, y la única que se encontró exclusivamente en 
dicha vegetación fue Selaginella tenella, colectada en la localidad ejido Santa María municipio de Casas.

Bosque de Pinus-Quercus: asociación vegetal que alberga 63 de las 91 especies citadas en el presente trabajo, de las cuales 24 taxones son estrictos de esta asociación vegetal, como son los casos de Anemia oblongifolia, Asplenium praemorsum, Astrolepis cochisensis, A. integerrima, Campyloneurum angustifolium, Cheilanthes angustifolia, Ch. arizonica, Ctenitis melanosticta, Dennstaedtia globulifera, Elaphoglossum sp., Lycopodiella cernua, Mildella fallax, Nephrolepis hirsutula, Osmunda regalis, Polypodium plebeium, Pteris biaurita, Selaginella novoleonensis, S. wrightii, Psilotum nudum, Thelypteris concinna, T. hispidula, T. melanochlaena, T. oligocarpa y T. puberula, colectados en las localidades de Rancho Briones, Arroyo San Miguel, Cerro San Miguel, Ejido el Plomo municipio de Aldama; Rancho Corazón de Jesús, Ejido Torrecillas, Cerro Torrecillas, Ejido Santa María, Las Pilas de Santa María, Cerro Eduardo Benavides, Ejido Eduardo Benavides, Rancho el Picacho, Valle de San José (cañón del diablo) municipio de Casas; Cerro Pedregoso, Cerro San Miguel municipio de González; Rancho los Venados municipio de Soto la Marina; Ejido el Chijol-Tuliyo, y San José (cañón del diablo) municipio de Llera.

Bosque de galería: en este tipo de vegetación las licofitas y monilofitas son relativamente escasas ya que únicamente se observó a Thelypteris ovata var. lindheimeri colectado en la localidad de Eslabones municipio de Soto la Marina.
Palmar: en la zona de estudio se encuentra con elementos de Quercus, aquí se recolectaron 21 especies de licofitas y monilofitas. Anemia sp., Cheilanthes brachypus, Ch. lozanoi var. seemannii, Ch. lozanoi vel. aff., Phlebodium decumanum fueron exclusivas de este tipo de vegetación propio del Rancho el Picacho, en el municipio de Casas.

\section{Similitud en la composición de especies por tipos de vegetación}

Se pueden definir tres grupos que indican la transición de las comunidades formando ecotonos. El primero corresponde a los bosques de Quercus y la asociación vegetal de bosque de Pinus-Quercus presentando la mayor distribución de las especies de licofitas y monilofitas; la siguiente mayor similitud está dada por el anterior grupo y la asociación de bosque Quercus y bosque tropical subcaducifolio; los anteriores grupos vegetales en su conjunto y la vegetación de palmar con Quercus a su vez muestran el tercer mayor valor de similitud. Una cuarta relación de similitud importante se da entre los anteriores tipos y asociaciones vegetales y el conformado por el matorral xerófilo y el bosque tropical subcaducifolio, los cuales a su vez muestran entre sí una similitud. El bosque de Pinus muestra una escasa relación con todos los anteriores tipos de vegetación, al igual que el bosque de galería el cual se muestra completamente diferente a los tipos de vegetación observados en este estudio, lo cual coincide con las altitudes geográficas de la zona (fig. 1). 


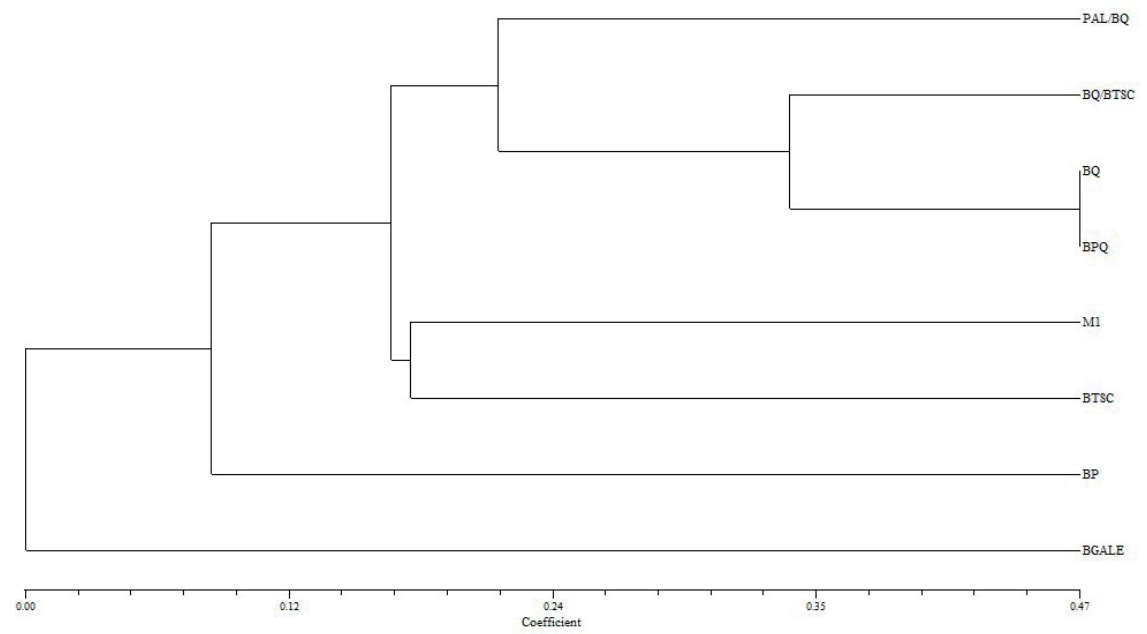

Fig. 1. Similitud en la composición de licofitas y monilofitas por tipos de vegetación en la sierra de Tamaulipas $(\mathrm{PAL} / \mathrm{BQ}=$ palmar con Quercus, BGALE = bosque de galería, $\mathrm{BQ} / \mathrm{BTSC}=$ bosque de Quercus con elementos de bosque tropical subcaducifolio, $\mathrm{MX}=$ matorral xerófilo, $\mathrm{BTSC}=$ bosque tropical subcaducifolio, $\mathrm{BP}=$ bosque de Pinus, $\mathrm{BQ}=$ bosque de Quercus, BPQ = bosque de Pinus-Quercus.

\section{Distribuciones altitudinales}

Se puede apreciar en el dendrograma de la figura 2, que la mayor parte de los pisos altitudinales en función de la diversidad de licofitas y monilofitas son diferentes. Se presentan fuertemente marcados dos grupos en los pisos altitudinales, uno de los 200 a los 400 m.s.n.m. que presentan 10 especies compartidas (10.19\%). El segundo piso altitudinal de los 400 a los $600 \mathrm{y}$ de los 600 a los 800 m.s.n.m., que son las más similares en su composición florística con un valor del índice de subordinación de 54\%. Los dos anteriores gradientes muestran mayor relación con el rango de 800-1000 m.s.n.m., y éstos a su vez mayor relación con el gradiente de 1000-1200 m.s.n.m.

\section{Distribución de especies por tipo de hábitat}

Terrestre: se observó un total de 72 especies, 47 de ellas se encuentran estrictamente en este sustrato como son los casos de Lycopodiella cernua, Selaginella hoffmannii, $S$. pallescens, Equisetum hyemale, Osmunda regalis, Lygodium venustum, Anemia sp., A. adiantifolia, A. hirsuta, A. karwinskiana, A. mexicana, Dennstaedtia globulifera, Pteridium caudatum, Adiantum concinnum, A. trapeziforme, A. tricholepis, A. tenerum, Llavea cordifolia, Pteris biaurita, Cheilanthes arizonica, Ch. brachypus, Ch. eatonii, Ch. kaulfussii, Ch. lozanoi var. seemannii, Ch. lozanoi sp., Cheiloplecton rigidum, Mildella fallax, M. intramarginalis, Notholaena candida, Astrolepis integerrima, Hemionitis palmata, Bomeria elegans, $B$. 
ISSN 2395-9525

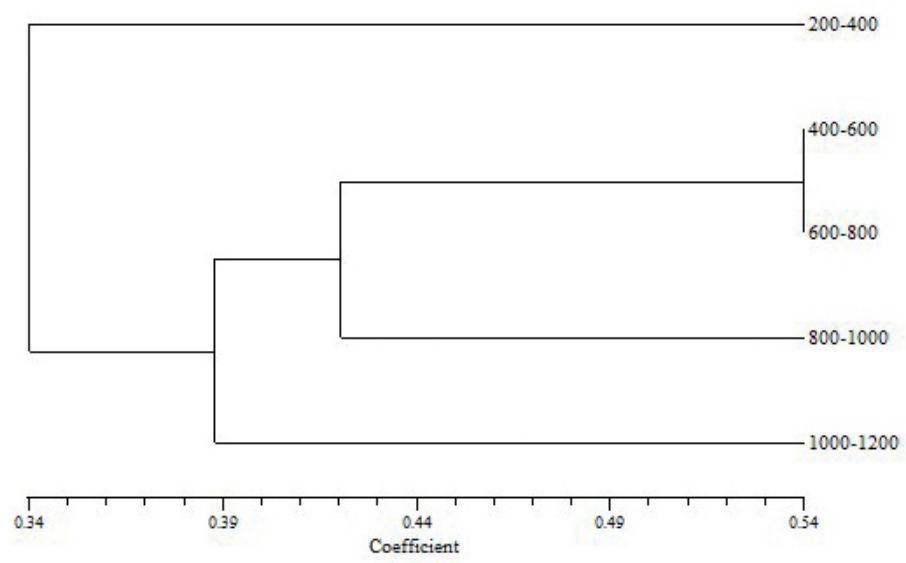

Fig. 2. Similitud de las especies por gradientes altitudinales en la sierra de Tamaulipas.

pedata, Asplenium monanthes, A. praemorsum, Thelypteris concinna, T. hispidula, T. melanochlaena, T. oligocarpa, T. ovata var. lindheimeri, T. puberula, Ctenitis melanosticta, Tectaria heracleifolia, Nephrolepis hirsutula, Campyloneurum angustifolium, Polypodium madrense, y P. plebeium.

Epipétrico o litofítico: de las 42 especies que fueron vistas en este hábitat, solamente 17 prefieren de forma exclusiva este sustrato, las cuales son: Selaginella delicatissima, S. extensa, S. hansenii, S. novoleonensis, $S$. tenella, S. wrightii, Psilotum complanatum, P. nudum, Trichomanes hymenophylloides, T. reptans, Anemia oblongifolia, Vittaria graminifolia, Astrolepis crassifolia, Pellaea ternifolia, Asplenium pumilum, Schaffneria nigripes, y Diplazium lonchophyllum.

Epifito: se observaron tres especies, solamente Phlebodium decumanum, se encontró exclusivamente en este sustrato, creciendo únicamente en el tronco de Sabal mexicana Mart. (Arecaceae).
Terrestres y epipétricos: Se encontraron 24 especies que comparten estos sustratos, tales son los casos de Selaginella lepidophylla, S. stenophylla, Adiantum capillus-veneris, Cheilanthes aemula, Ch. angustifolia, Ch. bonariensis, Ch. farinosa, Ch. leucopoda, Ch. microphylla, Ch. tomentosa, Aspidiotis meifolia, Notholaena brevistipes, Astrolepis cochisensis, A. sinuata, Pelleae ovata, Asplenium cuspidatum, A. minimum, Blechnum glandulosum, Elaphoglossum sp., E. sartorii, Phanerophlebia nobilis, Polypodium harwegianum, Phlebodium areolatum y Pecluma plumula.

Epipétrico y epifito: únicamente Pleopeltis mexicana comparte estos dos sustratos.

-Del total de las especies registradas únicamente Polypodium polypodioides se encontró en todos los sustratos. 
Hernández-Mendoza, A.M. et al.; Composición taxonómica de licofitas y monilofitas, sierra Tamps., Tamps., Méx.

\section{DisCUSIÓN Y CONCLUSIONES}

En trabajos anteriores como los de Gómez-Ruelas (1985) se citaron 76 especies de estas plantas vasculares para todo el estado de Tamaulipas; Johnston et al. (1989) mencionaron 80 especies para la sierra de Guatemala localizada en el municipio de Gómez Farías, Tamaulipas; Briones (1991) encontró 23 especies en la sierra de San Carlos, Tamaulipas; Martínez et al. (2004), enlistaron 92 especies de una porción de la Sierra Madre Oriental en Tamaulipas; Lof (2005) citó 64 especies de helechos de la sierra de Guatemala, en el área del Rancho El Cielo, Tamaulipas. Con los resultados de estos trabajos y con las especies colectadas para la sierra de Tamaulipas por Hernández-Mendoza (2007 y 2010), la pteridoflora de esta región contiene 91 taxones, lo cual representa el $65.4 \%$ de las 139 especies consideradas para el Estado de acuerdo con Mickel y Smith (2004).

De la flora Pteridológica presente en la zona sobresale la Familia Pteridaceae con 36 especies representada por 13 géneros, lo que equivale al $39.56 \%$ de las especies encontradas en este estudio, y con base en esta abundancia nuestros resultados concuerdan con los de Moran y Riba (1995) en el sentido de que esta familia es morfológicamente diversa, con más géneros que cualquier otra familia en Mesoamérica. El género que presentó más especies fue Cheilanthes con un total de 13 localizadas en diversos hábitat, lo cual coincide con Tryon y Tryon (1973), refiriéndose a que es un género numeroso con alrededor de 80 especies distribuidas en diferentes hábitats.

Las familias con más especies después de las Pteridaceae son Selaginellaceae y
Polypodiaceae, la primera representada por el género Selaginella con 10 especies, que según Moran y Riba (1995), el género se encuentra bien representado en bosques tropicales. Las Polypodiaceae en la zona de estudio cuenta con cinco géneros y nueve especies concentrándose la mayor diversidad en los bosques templados. En la Sierra de Tamaulipas se encontraron cuatro especies del género Polypodium.

Se presentan 23 taxones como nuevos registros para el Estado de Tamaulipas: Lycopodiella cernua, Selaginella hoffmannii, S. tenella, Psilotum complanatum, Osmunda regalis var. spectabilis, Trichomanes hymenophylloides, T. reptans, Anemia karwinskiana, A. oblongifolia, Pteris biaurirta, Cheilanthes arizonica, Ch. brachypus, Ch. eatonii, Ch. farinosa, Bommeria pedata, B. elegans, Diplazium lonchophyllum, Thelypteris concinna, T. melanochlaena, Elaphoglossum sartorii, Nephrolepis hirsutula, Polypodium madrense y Phlebodium decumanum.

Se cuenta con cinco taxones que pueden tratarse de nuevas especies como son: Selaginella hansenii vel. aff., Anemia sp., Cheilanthes eatonii vel. aff., Ch. lozanoi vel. aff., Elaphoglossum sp., cuyas características morfológicas no coinciden con ningún taxón descrito, además se encontró a Schaffneria nigripes, especie que no había sido recolectada en Tamaulipas por más de 30 años de acuerdo a Riba (1998).

Al revisar el trabajo de Mickel y Smith (2004) se encontró que de los estados del norte de la república mexicana, Chihuahua cuenta con 144 especies, le sigue Tamaulipas con 132, Nuevo León con 123, Sonora con 109 y Coahuila con 90. Con los aportes de la presente investigación se sumarían a las 
132 especies citadas por los autores referidos los 23 nuevos registros y las cinco posibles nuevas especies, sumando hasta el momento, un total de 160 especies para Tamaulipas convirtiéndola en la entidad federativa del norte de nuestro país con la mayor diversidad en licofitas y monilofitas.

La explicación de esta diversidad en la sierra de Tamaulipas se debe en gran parte a que en la zona de estudio convergen dos regiones, la Neártica y la Neotropical (Puig 1968), y también puede deberse a lo que menciona Tryon (1986), que en general las licofitas y monilofitas poseen una amplia distribución debido a que presentan características propias de dispersión y distribución.

Para el estado se cita una especie endémica que es Notholaena brevistipes misma que se encontró en la zona de estudio en las localidades: ejido Eduardo Benavides, cerro ejido Torrecillas, Las Pilas Santa María, Rancho Corazón de Jesús, Rancho el Picacho, Valle de San José, localidades del municipio de Casas; Rancho la Sauceda, cerro la Ventana localidades del municipio de González; ejido el Plomo, cerro San Miguel, Rancho Briones, ejido las Yucas localidades del municipio de Aldama; Rancho los Venados municipio de Soto la Marina; Francisco Castellanos, ejido el Chijol, la Lobera localidades del municipio de Llera. Dicho taxón fue colectado en vegetación de bosques de Quercus con elementos de bosque tropical subcaducifolio, matorral xerófilo, bosque de Quercus, bosque de Pino-Quercus, colectado en altitudes desde los 224 a los 1091 m.s.n.m.

La distribución de especies por tipos de vegetación que registró la mayor diversidad de taxones fue en la de Pinus-Quercus con el $68.13 \%$ de licofitas y monilofitas, lo que corresponde a 62 especies de las 91 colectadas. Según Rzedowski (1978) este tipo de comunidad corresponde a las que se distribuyen en climas templados y semihúmedos que se considera que favorece el crecimiento de estas criptógamas vasculares.

Según Tejero-Díez (2004), para el estado de Oaxaca menciona algunos taxones como Adiantum tenerum, Blechnum appendiculatum, Diplazium sp., Asplenium cuspidatum, A. praemorsum y algunas especies de los géneros Elaphoglossum y Polypodium que se encuentran bien representados en el soto bosque de los bosques mesófilos de montaña. En la Sierra de Tamaulipas dichas monilofitas fueron colectadas en el bosque tropical subcaducifolio, bosque de Pinus, Quercus, bosque de Pinus-Quercus ya que en el área de estudio no hay presencia de bosque mesófilo de montaña.

En cuanto al tipo de hábitat, el mayor número de especies de licofitas y monilofitas, prefieren el terrestre $(79.12 \%)$ y de ellos, estrictamente terrestres se tiene el 51.64\%. Posiblemente esta abundancia de especies se deba a que el suelo juega un papel fundamental en el soporte de los ecosistemas terrestres determinando su funcionamiento y productividad, lo anterior debido a la continua relación con el entorno litoclimático como el drenaje, la historia geomorfológica y el uso de la tierra según lo indica Cotler (2003), así también, Daubenmire (1999) indica que el suelo es un factor fundamental para el crecimiento de los organismos debido a que hay mayor cantidad de nutrientes y humedad, además le proporciona fijación a la planta y existe una relación estrecha con el clima. Arreguín-Sánchez et al. (2009a) indican que los helechos y plantas afines 
Hernández-Mendoza, A.M. et al.; Composición taxonómica de licofitas y monilofitas, sierra Tamps., Tamps., Méx.

juegan un papel ecológico muy importante por ser indicadores de la salud de los ecosistemas debido a la sensibilidad que tiene la fase gametofítica a los cambios en las condiciones microambientales.

De las especies citadas para la sierra de Tamaulipas, el piso altitudinal de 400 a 1200 m.s.n.m. mostró mayor representatividad de especies, ya que ahí fueron recolectadas 81 taxones de licofitas y monilofitas de las 91 registradas para el área, lo que representa el $89.01 \%$, del total de las especies. Esta preferencia en la distribución de las licofitas y monilofitas está fuertemente relacionada con los tipos de vegetación presentes en el área de estudio como son los bosques de Quercus y Pinus-Quercus que presentan mayores condiciones de humedad, además de presentar árboles que pueden sustentar a las especies epífitas y la protección directa de los rayos solares. Las 10 especies restantes corresponden al $10.99 \%$ del total de las especies y se encuentran distribuidas en el piso altitudinal de los 200 a los 400 m.s.n.m. lo que corresponde a las partes bajas de la sierra, y por lo tanto, donde existe menos humedad para el desarrollo de este grupo de plantas.

Algunas de las especies encontradas en áreas con disturbios fueron Lycopodiella cernua y Osmunda regalis creciendo en suelos pantanosos, áreas perturbadas de bosques abiertos de Pinus-Quercus donde se observa la presencia de actividad humana. Otras de las especies que se encontraron en laderas, caminos y potreros fueron Asplenium minimum, Blechnum appendiculatum y Pteridium caudatum, esta última considerada indicadora de disturbio. Cabe mencionar que en este estudio el $79.12 \%$ de las licofitas y monilofitas prefieren el sustrato terrestre, por lo anterior hay que resaltar la importancia de tomar medidas urgentes para la protección de estos hábitat para evitar su fragmentación.

En cuanto al estatus de conservación y de acuerdo a la Norma Oficial Mexicana (NOM-ECOL-059-2002), Psilotum complanatum se encuentra en la categoría de amenazada, que de acuerdo a la norma, son aquellas especies que pueden llegar a desaparecer a corto o mediano plazo si siguen operando factores que inciden negativamente. Otro de los casos para el área de estudio es el de Schaffneria nigripes, la cual no había sido recolectada desde hace 30 años según Riba (1998). Hernández-Mendoza (2007) indica que dicha especie podría considerarse una planta en peligro de desaparecer en la zona debido a que su crecimiento se restringe a la localidad ejido Torrecillas, distribuyéndose en las cañadas que sustentan un bosque tropical subcaducifolio que se encuentra muy devastado debido a la presencia de asentamientos humanos. Arreguín et al. (2009b) mencionan, en un estudio enfocado a las licofitas y monilofitas extintas o raras, que algunos factores como la actividad humana han traído consigo la extinción e introducción de especies, ocasionando cambios en el funcionamiento de los ecosistemas que pueden conllevar a la extinción de especies al presentar una distribución discontinua o muy limitada, lo cual ocurre para el área de estudio con las siguientes especies: Lycopodiella cernua, Selaginella hansenii, S. hoffmannii, S. novoleonensis, S. pallescens, S. tenella, Osmunda regalis, Trichomanes hymenophylloides, T. reptans, Anemia sp., A. oblongifolia, Vittaria graminifolia, Pteris biaurita, Cheilanthes 
brachypus, Ch. eatonii vel. aff., Ch. lozanoi var. seemannii, Ch. lozanoi vel. aff., Astrolepis cochisensis, Pellaea ternifolia, Bommeria elegans, B. pedata, Asplenium praemorsum, Diplazium lonchophyllum, Thelypteris melanochlaena, Elaphoglossum sp., E. sartorii, Nephrolepis hirsutula, y Phlebodium decumanum.

\section{LITERATURA CITADA}

Arreguín-Sánchez, M.L.; R. Fernández-Nava, D.L. Quiroz-García, y S. Acosta-Castellanos, 2009a. "Análisis de la distribución de las especies de helechos y afines del Valle de México". Polibotánica. 28: 15-36.

Arreguín-Sánchez, M.L., D.L. Quiroz-García, y R. Fernández-Nava, 2009b. "Pteridofitas extintas o raras del Valle de México". Polibotánica. 27: 17-29.

Arriaga, L., J.M. Espinoza, C. Aguilar, E. Martínez, L. Gómez y E. Loa (coordinadores), 2000. Regiones terrestres prioritarias de México. Comisión Nacional para el Conocimiento y Uso de la Biodiversidad, México. ,p. 377-379.

Briones, O. L., 1991. "Sobre la flora, vegetación y fitogeografía de la Sierra de San Carlos, Tamaulipas". Act. Bot. Mex., 16: 15-43.

Christenhusz, M.J.M.; X. Chun Z. y H. Schneider, 2011. "A linear sequence of extant families and genera of lycophytes and ferns". Phytotaxa, 19: 7-54.

Comisión Nacional de Áreas Naturales Protegidas. 2006. Estudio Previo
Justificativo para el establecimiento de la Reserva de la Biosfera Sierra de Tamaulipas. México, DF, 89 pp. www.conanp.gob.mx/anp/consulta/ EPJ-Sierra.pdf

Cotler, A.H., 2003. "Características y Manejo de suelos en Ecosistemas Templados". Conservación de ecosistemas Templados de Montaña en México. Sánchez O.; E. Vega, E. Peters, y O Monroy-Vilchis (editores). Instituto Nacional de Ecología. México. 316 pp.

Daubenmire, R.F., 1999. Ecología Vegetal, Tratado de Autoecología de Plantas. Editorial Limusa, S.A. de C.V. México, DF. 496 pp.

Diario Oficial de la Federación, 2002. Norma oficial mexicana NOM-059-SEMARNAT-2001. "Protección ambiental-Especies nativas de México de flora y fauna silvestres-Categorías de riesgo y especificaciones para su inclusión, exclusión o cambio-Lista de especies en riesgo". 6 de marzo. Primera sección. 85 pp.

Gómez-Ruelas, N.H., 1985. Contribución al conocimiento Florístico de las pteridofitas en el estado de Tamaulipas, México. Tesis de licenciatura en Ciencias Biológicas. Universidad del Noreste, Tampico, Tamaulipas, 116 pp.

Hernández-Mendoza, A.M., 2007. "Flora Pteridológica de la Parte Sur de la Sierra de Tamaulipas, Tamaulipas, México". Tesis de licenciatura. Instituto Tecnológico de Ciudad Victoria Tamaulipas. 117 pp. 
Hernández-Mendoza, A.M., 2010. “Composición Taxonómica y Fitogeografia de las Pteridofitas de la Sierra de Tamaulipas, Tamaulipas, México". Tesis de maestría en Ciencias en Biología, con Especialidad en Manejo y Conservación de Recursos Naturales. Instituto Tecnológico de Ciudad Victoria Tamaulipas. $191 \mathrm{pp}$.

Instituto Nacional de Estadística, Geográfica e Informática, 1983. Sintesis Geográfica del estado de Tamaulipas, México DF, 157 pp.

Johnston, M.C.; K. Nixon, y G. L. Nesom. 1989. "Listado de plantas vasculares conocidas de la sierra de Guatemala, Gómez Farías, Tamaulipas, México". Biotam., 1(2): 21-33.

Lof, L.V., 2005. "A preliminary list of pteridophytes from the eastern slope of the sierra de Guatemala". 209-211 pp. en Historia natural de la Reserva de la Biosfera el Cielo, Tamaulipas, México (Sánchez, G., P. Ceballos, y R. Dirzo, eds), Universidad Autónoma de Tamaulipas. Impreso en Hong Kong. 732 pp.

Martin, P.S.; C. Richard R., y B.H. William, 1954. "Birds and biogeography of the sierra de Tamaulipas, an isolated pine-oak habitat". The Willson Bulletin., 66: 1-38.

Martínez, M.; L. Hernández-Sandoval, A. Mora O., y A. Domínguez-Monroy. 2004. "Florística de Tamaulipas". 215-242 pp. en: Biodiversidad de la Sierra Madre Oriental. (Luna, I.; J.J. Morrone, y D. Espinosa, eds.). Comisión Nacional para el Conocimiento y Uso de la Biodiversidad, Universidad Nacional Autónoma de México, Las Prensas de Ciencias, UNAM, México, DF, 527 pp.

Mickel, J.T., y J. M. Beitel. 1988. "Pteridophyte flora of Oaxaca, México". Mem. New York Bot. Gard., 46: 1-568.

Mickel, J.T., y A.R. Smith, 2004. "The pteridophytes of Mexico". Mem. New York Bot. Gard., 88: 1-1054.

NTSYSpc, versión 2.02. Copyright (C) 1986. Applied Biostatistics Inc., all Rights reserved worldwide.

Moran, R.C., y R. Riba (eds.), 1995. "Psilotaceae a Salviniaceae". In Davidse, G., M. Sousa y S. Knapp (eds. grales). Flora Mesoamericana. Instituto de Biología, Universidad Nacional Autónoma de México-Missouri Botanical Garden-The Natural History Museum (Londres), México, DF, 1: 1-470.

Puig, H., 1968. "Notas de la Flora y Vegetación de la Sierra de Tamaulipas (México)" Anales. Escuela Nacional de Ciencias Biológicas, México. 17: 37-49.

Riba, R., 1998. "Pteridofitas mexicanas: distribución y endemismos", en $D i$ versidad Biológica de México: $369-$ 384. Ramamoorthy, T.P. et al., (eds) 
Instituto de Biología, Universidad Nacional Autónoma de México.

Rzedowski, J., 1978. Vegetación de México. Ediciones Limusa. México, DF. 432 pp.

Tejero-Díez, J.D., y J.T. Mickel, 2004. "Pteridofitas". 121-129 pp., en: Biodiversidad de Oaxaca (García-Mendoza, A.J.. M.J. Ordónez, y M. Briones-Salas, eds.). Instituto de Biología, UNAM-Fondo Oaxaqueño para la Conservación de la Naturaleza-World Wildlife Found. México. 605 pp.
Tryon, R.M., y A.F. Tryon, 1973. "Geography, spores and evolution in the Cheilanthes ferns", Bot. Linn. Soc. (Suppl.), 67: 145-153. , 1982. Ferns and allied plants, with special reference to tropical America. Springer-Verlag. New York. 857 pp.

Tryon, R.M., 1986. "The biogeography of species, with special reference to ferns". Botanical Review, 52(2): 117-155.

Recibido: 30 octubre 2013. Aceptado: 21 febrero 2014. 